\title{
Screening for 392 polymorphisms in 141 pharmacogenes
}

\author{
JASON YONGHA KIM ${ }^{1 *}$, HYUN SUB CHEONG ${ }^{2 *}$, TAE-JOON PARK ${ }^{1}$, HEE JUNG SHIN ${ }^{3}$, \\ DOO WON SEO ${ }^{3}$, HAN SUNG NA ${ }^{3}$, MYEON WOO CHUNG ${ }^{3}$ and HYOUNG DOO SHIN ${ }^{1,2}$ \\ ${ }^{1}$ Department of Life Science, Sogang University; ${ }^{2}$ Department of Genetic Epidemiology, SNP Genetics, Inc., \\ Seoul 121-742; ${ }^{3}$ Division of Clinical Reaserch, Department of Toxicological Evaluation and Research, \\ National Institute of Food and Drug Safety Evaluation, Osong Health Technology Administration Complex, \\ Osong, Chungcheongbuk 363-700, Republic of Korea
}

Received February 17, 2014; Accepted March 28, 2014

DOI: $10.3892 / b r .2014 .272$

\begin{abstract}
Pharmacogenomics is the study of the association between inter-individual genetic differences and drug responses. Researches in pharmacogenomics have been performed in compliance with the use of several genotyping technologies. In this study, a total of 392 single-nucleotide polymorphisms (SNPs) located in 141 pharmacogenes, including 21 phase I, 13 phase II, 18 transporter and 5 modifier genes, were selected and genotyped in 150 subjects using the GoldenGate assay or the SNaPshot technique. These variants were in Hardy-Weinberg equilibrium (HWE) $(\mathrm{P}>0.05)$, except for 22 SNPs. Genotyping of the 392 SNPs revealed that the minor allele frequencies of 47 SNPs were $<0.05,105$ SNPs were monomorphic and 22 variants were not in HWE. Also, based on previous studies, we predicted the association between the polymorphisms of certain pharmacogenes, such as cytochrome P450 2D6, cytochrome P450 2C9, vitamin K epoxide reductase complex, subunit 1, cytochrome P450 2C19, human leukocyte antigen, class I, B and thiopurine S-methyltransferase, and drug efficacy. In conclusion, our study demonstrated the allele distribution of SNPs in 141 pharmacogenes as determined by high-throughput screening. Our results may be helpful in developing personalized medicines by using pharmacogene polymorphisms.
\end{abstract}

\section{Introduction}

Inter-individual variation in drug response among patients is a major obstacle in medicine application, due to the different response of each patient to the same medication (1). Several cases of adverse drug reactions occurring in certain patients,

Correspondence to: Professor Hyoung Doo Shin, Department of Life Science, Sogang University, 1 Shinsu-dong, Mapo-gu, Seoul 121-742, Republic of Korea

E-mail: hdshin@sogang.ac.kr

*Contributed equally

Key words: gene screening, pharmacogene, single-nucleotide polymorphism such as renal/hepatic disorders, congestive heart failure and anemia, were previously reported $(1,2)$. The variations in drug responses may result from disease determinants, genetics, environmental factors and idiosyncratic response, which collectively affect drug metabolism (3). The knowledge of variations in efficacy and toxicity caused by the same doses of medications may enhance the effectiveness of drug therapy (3).

The initial human genome sequencing identified $\sim 1.42$ million single-nucleotide polymorphisms (SNPs), including $>60,000$ SNPs in the exonic region of genes (4). Some of these SNPs have been suggested to be associated with considerable changes in drug disposition and metabolism or the effects of medication (5-7), whereas others are used for the diagnosis of clinical response (8). The interaction of several gene products is known to affect the pharmacokinetics and pharmacodynamics of medications. For example, inherited variations in drug targets, drug disposition and polygenic factors of drug effects are determinants of the majority of drug effects and have become increasingly important in pharmacogenomics (9).

Pharmacogenomics is the study of how genetic differences among individuals affect the variability in their response to medications $(6,10)$. Pharmacogenomic research includes clinical and basic science regarding genetic variation and drug response. The field of pharmacogenomics has attracted significant attention along with the completion of the Human Genome Project (11). Consequently, there has been an increase in the number of pharmacogenomic studies published (11). Furthermore, the continuous development of genotyping technologies may allow pharmacogenomic applications to move into the mainstream of medicine and pharmacy practice $(12,13)$.

Over the last few years, the available technologies for genomic analyses have increased significantly (14). For example, the TaqMan and the SNPlex assays from Applied Biosystems (Foster City, CA, USA), the GeneChip assay from Affymetrix (Santa Clara, CA, USA) and the Infinium and GoldenGate assays developed by Illumina (San Diego, CA, USA) are the most frequently used techniques in genome research (15). In addition, the SNaPshot technique has been tested on a small scale of multiplex for analysis of gene polymorphisms (16). In this study, we used the GoldenGate assay and the SNaPshot technique for genotyping to investigate the 
allele distribution of 392 SNPs from 141 pharmacogenes in 150 Korean subjects.

\section{Materials and methods}

Study subjects. DNA samples from a total of 150 Korean subjects was used for this study. The 150 unrelated Korean samples were provided by the Center for Genome Science, Korea Centers for Disease Control and Prevention. The protocol and consent forms of this study were reviewed and approved by the Institutional Review Board of Sogang University (no. 2010_690).

SNP selection and genotyping. We selected a total of 378 SNPs of 141 well-known pharmacogenes, based on the score assigned by the Illumina GoldenGate assay design tool (ADT; Illumina). The SNPs were genotyped using the GoldenGate assay with the VeraCode microbead (Illumina) $(17,18)$, followed by a scan using the BeadExpress ${ }^{\circledast}$ system (Illumina). Normalized bead intensity data obtained for each sample were loaded into the GenomeStudio ${ }^{\circledR}$ software (Illumina), which converted fluorescent intensities into SNP genotypes. SNP clusters for genotype calling were examined for all SNPs using the GenomeStudio ${ }^{\circledR}$ software. The cluster plots were then visually assessed and SNPs with poor cluster quality were removed. The overall call rate for all SNPs was $99.99 \%$. For quality control, SNPs that met the following criteria were retained: call rate $\geq 0.98 \%$ and no triplicate error after three repetition tests. Fourteen additional SNPs which did not pass ADT scoring were genotyped using the SNaPshot technique (Invitrogen Life Technologies, Carlsbad, CA, USA). The SNaPshot technique is single- extension-based method, which enables the simultaneous analysis of multiple SNPs (19). The information of SNaPshot primers used for the 14 SNPs are shown in Table I. The GoldenGate and $\mathrm{SNaPshot}$ assays were conducted three times in order to increase the accuracy of the test.

Statistical analysis. The Chi-square test was used to determine whether individual variants were in Hardy-Weinberg equilibrium (HWE) at each locus in a Korean population. Haplotypes of SNPs representing the star-alleles of each pharmacogene investigated in this study were defined using PHASE software (Stephen Laboratory, University of Chicago, Chicago, IL, USA).

\section{Results and Discussion}

A total of 392 SNPs located in 141 pharmacogenes (including 21 phase I, 13 phase II, 18 transporter and 5 modifier genes) were successfully genotyped in 150 Korean subjects and their minor allele frequencies (MAFs) were calculated (Table II). These variants were in HWE ( $\mathrm{P}>0.05)$, except for $22 \mathrm{SNPs}$. Among the SNPs, 47 variants exhibited a MAF $<0.05$, while 105 variants exhibited a monomorphic allele distribution in the Korean population (Table III). In addition, there were 22 SNPs with $\mathrm{HWE}<0.05,9$ of which exhibited significantly lower HWE compared to others [rs2230037, rs2472393, rs 743544 [all in the glucose-6-phosphate dehydrogenase $(G 6 P D)$ gene], rs2227291 [copper-transporting ATPase 1 (ATP7A) gene], rs 1414334, rs518147, rs3813928, rs6318 and rs3813929 [all in the 5-hydroxytryptamine receptor $2 \mathrm{C}(H T R 2 C)$ gene]. The
3 genes were all located in the $\mathrm{X}$ chromosome, although located far away from each other (G6PD at Xq28, ATP7A at Xq21.1 and $H T R 2 C$ at Xq24). The classifications and SNP numbers of each pharmacogene investigated in this study are listed in Table IV. Among these, cytochrome P450 2D6 (CYP2D6), cytochrome P450 2C19 (CYP2C19), thiopurine S-methyltransferase (TPMT), cytochrome P450 2C9 (CYP2C9), vitamin K epoxide reductase complex, subunit 1 (VKORCl) and human leukocyte antigen, class I, B (HLA-B) are of great significance, due to their association with well-known drugs. Therefore, we investigated the respective genes and their effects on enzyme activity or associated drugs below.

First, 14 CYP2D6 SNPs (rs1065852, rs16947, rs1135822, rs35742686, rs3892097, rs5030655, rs79738337, rs1058164, rs1135840, rs28371525, CYP2D6_2, rs5030867, rs5030865 and $r s 5030656$ ) were used for the investigation of 17 star-alleles $\left({ }^{*} 1,{ }^{*} 2,{ }^{*} 3,{ }^{*} 4,{ }^{*} 5,{ }^{*} 6,{ }^{*} 7,{ }^{*} 8,{ }^{*} 9,{ }^{*} 10,{ }^{*} 14 A,{ }^{*} 14 B,{ }^{*} 34,{ }^{*} 36, " 41,{ }^{*} 49\right.$ and "60). CYP2D6 is one of the most important pharmacogenes involved in the metabolism of foreign substances in the body. Overall, the genotypes associated with decreased activity or a non-functional enzyme were $20 \%$ of all the investigated genotypes (Table V). Specifically, screening of polymorphisms such as $r s 3892097, r s 1065852, r s 16947$ and rs1135840 may be useful for detecting the enzyme activity level of CYP2D6. CYP2C19 is clinically important for the metabolism of drugs including clopidogrel (20), which is an inhibitor of adenosine diphosphate-induced platelet aggregation (21-23). To investigate the association between $C Y P 2 C 19$ and clopidogrel response, $15 C Y P 2 C 19$ SNPs were used for the investigation of seven alleles $\left(" 1,{ }^{*} 2,{ }^{*} 3,{ }^{*} 4,{ }^{*} 5 A,{ }^{*} 8\right.$ and $\left.{ }^{*} 17\right)$ (Table V). In general, over half of the subjects were found to have genotypes that reduced the efficacy of clopidogrel, while $11.5 \%$ of the subjects carried genotypes which enhanced the efficacy of clopidogrel. This information may be used to adjust the dose of clopidogrel depending on the patient genotypes of the investigated polymorphisms.

$T P M T$ encodes an enzyme involved in the detoxification of azathioprine, mercaptopurine and thioguanine, which are immunosuppressive drugs used in organ transplantation (24-27). Five SNPs (rs75543815, rs1142345, rs1800460, $r s 1800462$ and $r s 1800584)$ were used to investigate the frequency of five alleles $\left({ }^{*} 2,{ }^{*} 3 B,{ }^{*} 3 C,{ }^{*} 4\right.$ and $\left.{ }^{*} 6\right)$ in this study (Table V). The results suggested that the majority of Korean subjects have TPMT genotypes, which render them more prone to the toxicity of the aforementioned drugs. The dihydropyrimidine dehydrogenase gene $(D P Y D)$ encodes an enzyme catabolizing 5-fluorouracil (5-FU), which is commonly used for the treatment of solid carcinomas $(28,29)$. A decrease in enzyme activity involved in 5-FU catabolism due to the mutational variants in DPYD may lead to an increase in the half-life of 5-FU and an increased risk of dose-dependent toxicity $(28,30,31)$. In our study, all the Korean subjects were found to carry the DPYD genotypes associated with normal enzyme activity (" $1 /{ }^{*} 5,{ }^{*} 5 /{ }^{*} 5,{ }^{*} 1 /{ }^{*} 9 A$, or $c .496 A>G$; Table V). A polymorphism of interleukin 28B (IL28B), rs8099917 has been reported to be associated with the virologic response to peginterferon- $\alpha$ (PEG-IFN $\alpha$ ) and ribavirin (RBV) combination therapy in hepatitis $\mathrm{C}$ virus-infected patients (32-34), whereas the GT and GG genotypes of $r s 8099917$ have been suggested to be less responsive to treatment compared to 
Table I. SNaPshot primer sequences for 14 single-nucleotide polymorphisms (SNPs).

\begin{tabular}{|c|c|c|c|}
\hline Gene & SNP & Primer & Sequence \\
\hline \multirow[t]{10}{*}{$C Y P 2 D 6$} & & Forward & GTTATCCCAGAAGGCTTTGCAGGCTTCA \\
\hline & & Reverse & GCCGACTGAGCCCTGGGAGGTAGGTA \\
\hline & $\operatorname{rs} 1065852^{\mathrm{a}}$ & SNaPshot & AACGCTGGGCTGCACGCTAC \\
\hline & $\operatorname{rs} 16947^{\mathrm{a}}$ & SNaPshot & TCAGAGAACAGGTACCACCACTATGC \\
\hline & $r s 1135822^{\mathrm{a}}$ & SNaPshot & T(11)CGGGCCCATAGCGCGCCAGGA \\
\hline & $r s 35742686^{\mathrm{a}}$ & SNaPshot & T(10)CCAGCTGGATGAGCTGCTAACTGAGCAC \\
\hline & $r s 3892097^{\mathrm{a}}$ & SNaPshot & T(20)CCTTACCCGCATCTCCCACCCCCA \\
\hline & $r s 5030655^{\mathrm{a}}$ & SNaPshot & T(24)GCCTGGGCAAGAAGTCGCTGGAGCAG \\
\hline & $\operatorname{rs} 79738337^{\mathrm{a}}$ & SNaPshot & T(31)GGCAAGGAGAGAGGGTGGAGGCTGG \\
\hline & $\operatorname{rs} 1058164^{\mathrm{a}}$ & SNaPshot & T(41)CGAGCAGAGGCGCTTCTCCGT \\
\hline \multirow[t]{3}{*}{$D P Y D$} & $r s 72981743$ & Forward & CGAAAACAGGCAGACTAGGG \\
\hline & & Reverse & AGAGCGGGTGCTCTACTCC \\
\hline & & SNaPshot & TCTGCTTGCAGGCTGGGGCGC \\
\hline \multirow[t]{3}{*}{ CYP2A6 } & $r s 56256500$ & Forward & AGTTGGCAGGTTGTGGTAGG \\
\hline & & Reverse & CTCCAATGTCATCAGCTCCA \\
\hline & & SNaPshot & GAACTGGAAGATTCCTAGCATCATGC \\
\hline \multirow[t]{3}{*}{ NRII2 } & rs 1464603 & Forward & CACCAGCCCACACTCTGAAC \\
\hline & & Reverse & CAAATCTGCCGTGTATGTGG \\
\hline & & SNaPshot & CTGGGGGACAGGTCAAGCTGAGGCCCTGAGA \\
\hline \multirow[t]{3}{*}{ CYP2A6 } & rs 1809810 & Forward & TCCAGCCCCTGTGTACTTTC \\
\hline & & Reverse & AAACTGCCCCTTCTCATTCA \\
\hline & & SNaPshot & T(7)CAACTTCCTCCTCCCTACCAGGGCACCGAAGTGT \\
\hline \multirow[t]{3}{*}{$A B C B 1$} & $r s 2032582$ & Forward & TTGAAATGAAAATGTTGTCTGGA \\
\hline & & Reverse & AAAAGATTGCTTTGAGGAATGG \\
\hline & & SNaPshot & T(13)CAAGCACTGAAAGATAAGAAAGAACTAGAAGGT \\
\hline \multirow[t]{3}{*}{ CYP2C19 } & $r s 3758580$ & Forward & TTCATGTACCCCTGAATTGCT \\
\hline & & Reverse & CATCTGTGTAGGGCATGTGG \\
\hline & & SNaPshot & T(24)GCATGCAGGGGCTCCGGTTTCTGCCAAC \\
\hline
\end{tabular}

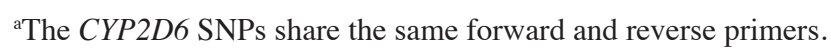

TT (32). In this study, subjects carrying the TT genotype were the most common (86.7\%), whereas the GT and GG genotypes were significantly less frequent (12.7 and $0.7 \%$, respectively) in the Korean population (Table V). These results may be used to identify patients with reduced responsiveness to PEG-IFN $\alpha /$ RBV combined therapy and adjust the amount accordingly.

Warfarin is an anticoagulant used for the prevention of thromboembolic events and stroke (35). CYP2C9*2 variants, *3 variants (36-40) and VKORC1 polymorphism rs 8050894 (41) were reported to be significantly associated with warfarin dose in European or African populations. In this study, we evaluated the variability of warfarin dose according to the $C Y P 2 C 9$ and VKORC1 genotypes using CYP2C9*2, *3 and rs8050894, based on previous reports (Table VI). Combinations of the CG or CC genotype of rs8050894 and CYP2C 9 wild-type $\left({ }^{*} 1 /^{*} 1\right)$ yielded the highest warfarin dose requirement $(5-7 \mathrm{mg} /$ day), whereas a combination of the GG genotype of $r s 8050894$ and CYP $2 C 9^{*} 1 /^{*} 1$, demanding 3-4 mg/day of warfarin, was the most frequent $(76.0 \%)$ in the Korean population. Furthermore, the warfarin dose requirement in $C Y P 2 C 9$ wild-type $\left({ }^{*} 1 /^{*} 1\right)$ was higher compared to that in *2 or *3 variant allele-containing genotypes $\left(* 1 / /^{*} 2,{ }^{*} 1 / /^{*} 3,{ }^{*} 2 /^{*} 2,{ }^{*} 2 /^{*} 3\right.$ and $\left.{ }^{*} 3 /{ }^{*} 3\right)$, which was also observed in European and African-American populations (42). As regards $r 8050894$ in $V K O R C 1$, an overall higher warfarin dose requirement in $\mathrm{CG}$ (heterozygote) or CC (minor homozygote) compared to that in GG (major homozygote) was observed. However, in European and African-American populations, GG exhibited a higher warfarin dose requirement compared to the CG or CC genotype (42). Further investigations may be required to verify the different genetic effect on warfarin response in various ethnic groups.

$H L A-B$ encodes for a protein which is an important part of the human immune system and its polymorphisms have been associated with various drug reactions (43-45). Carbamazepine, which is often used for treatment of chronic pain, bipolar disorder, seizure disorder and trigeminal neuralgia, is one of the most common causes of drug hypersensitivity reactions (46). The star-allele $H L A-B^{*} 1502$ is associated with various toxic events resulting from carbamazepine, such as cutaneous 


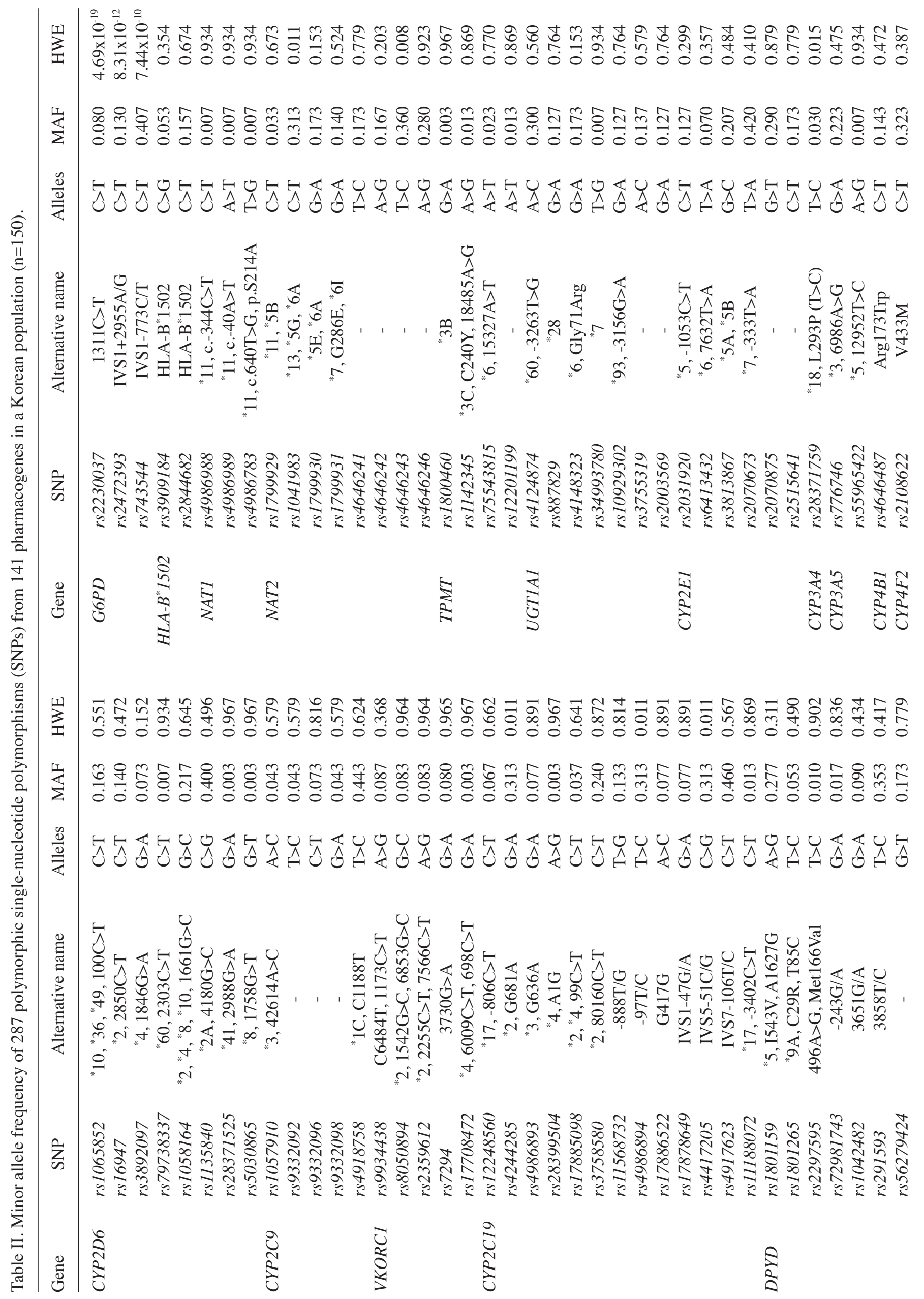




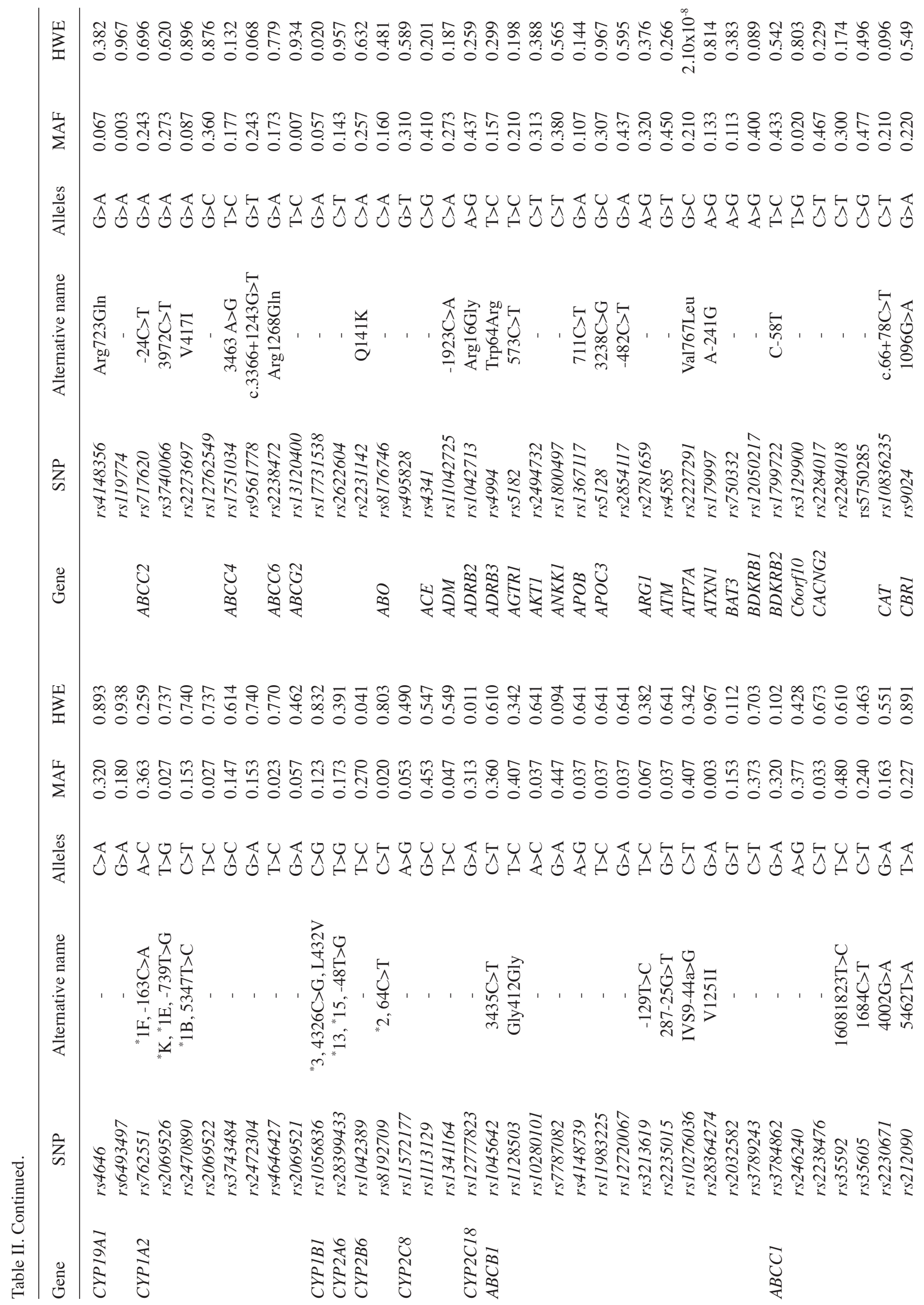




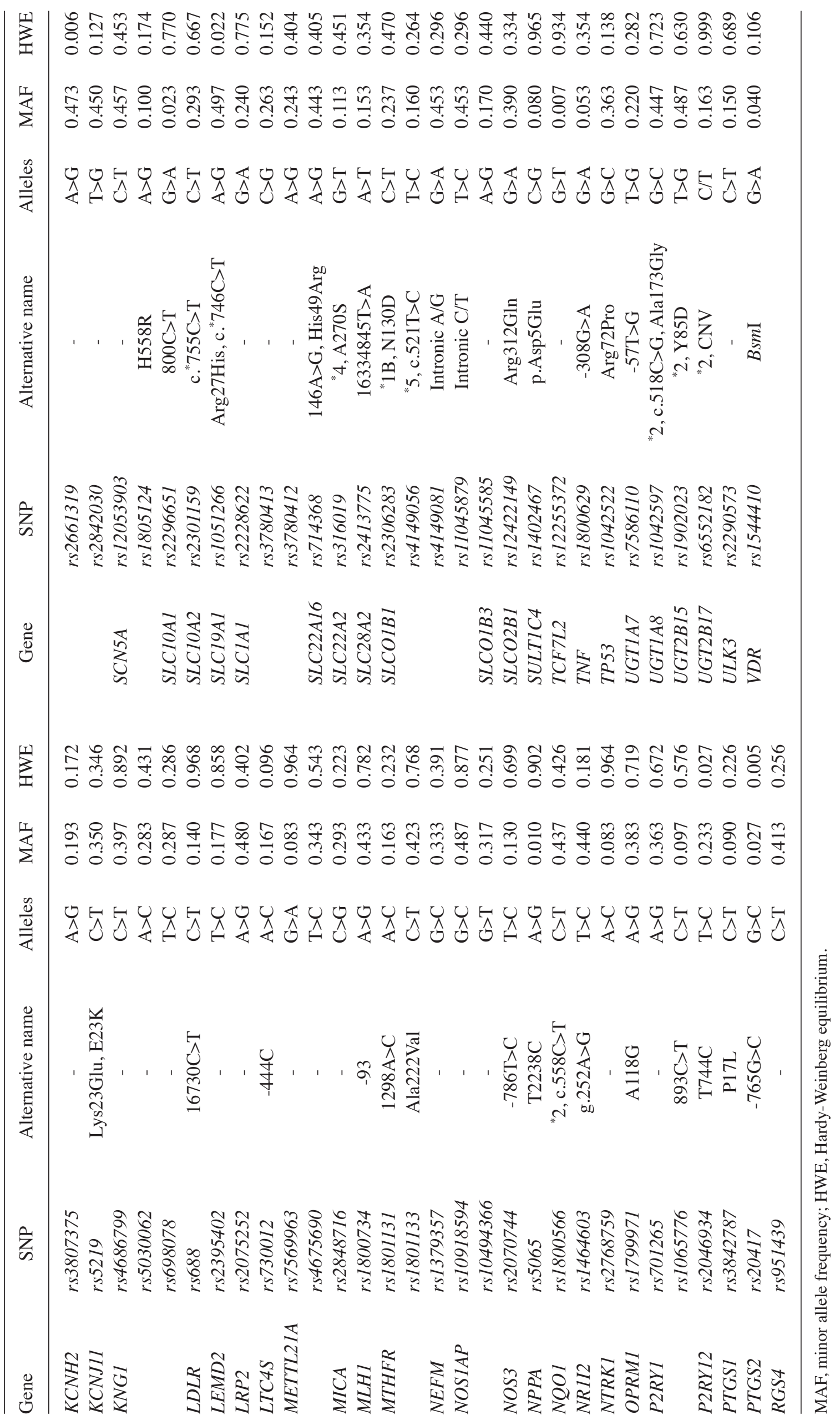




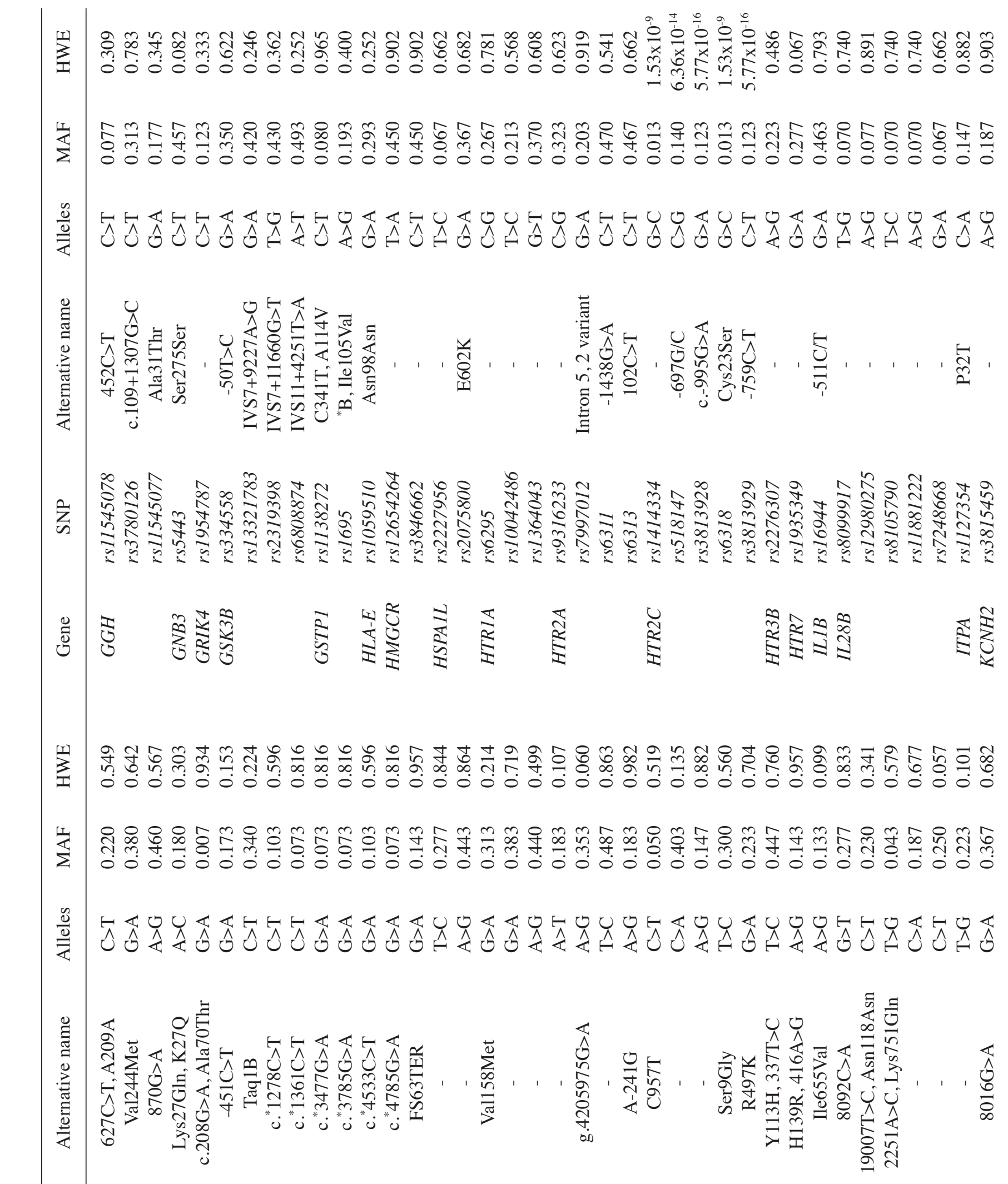

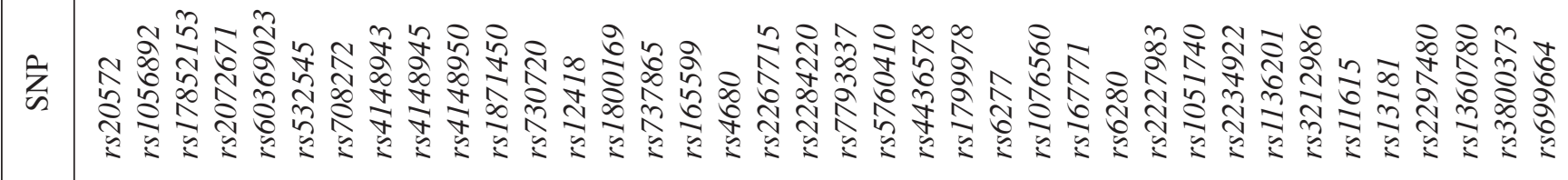

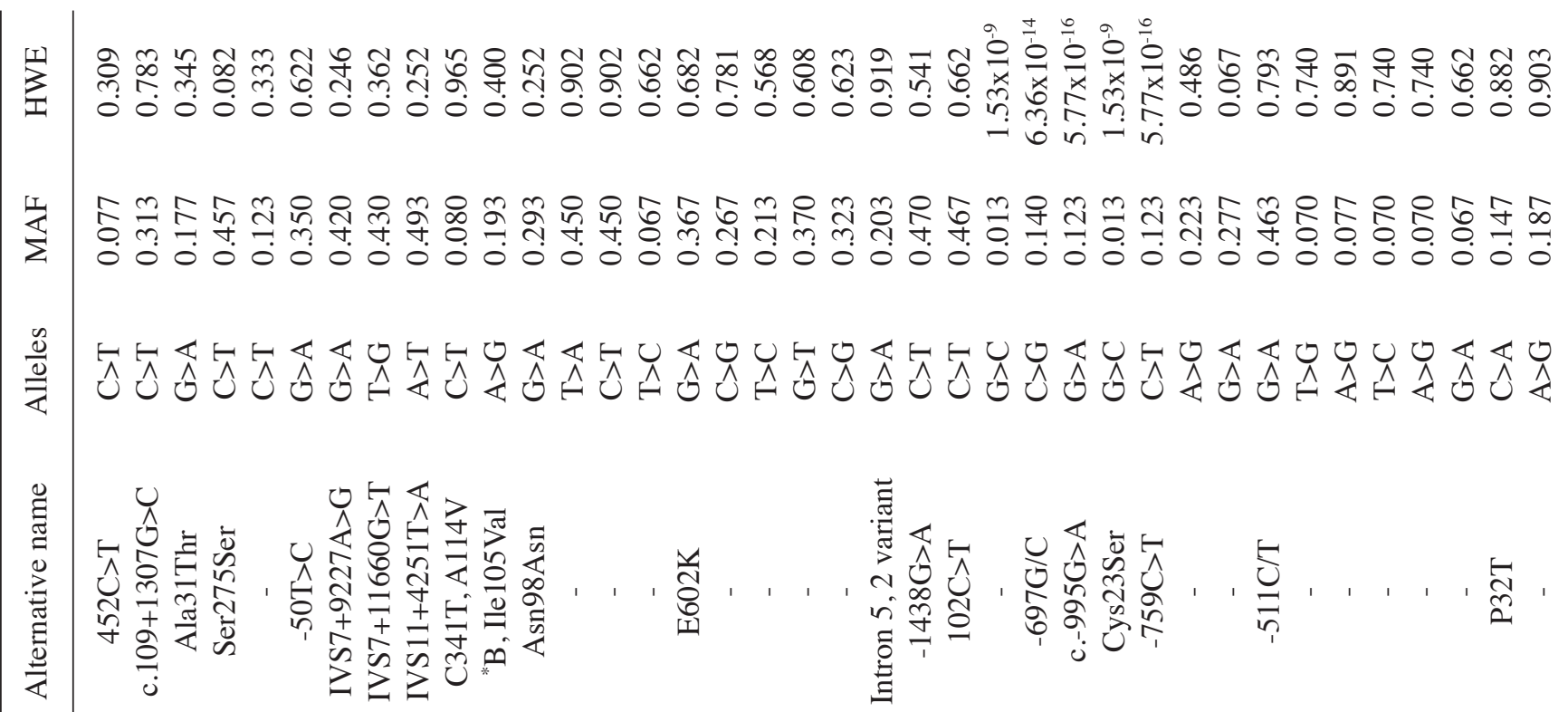

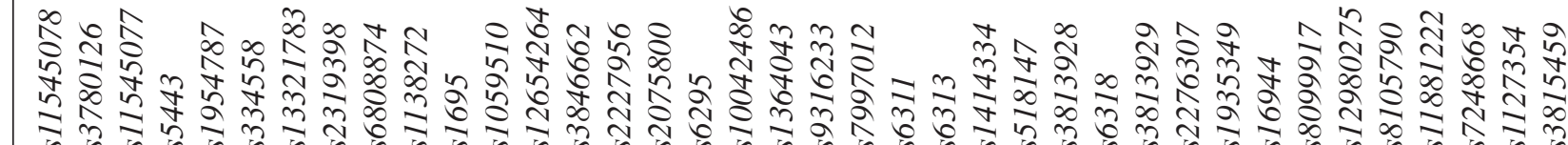

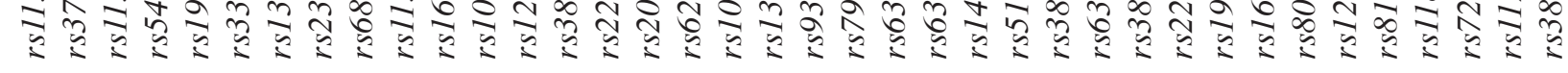

范

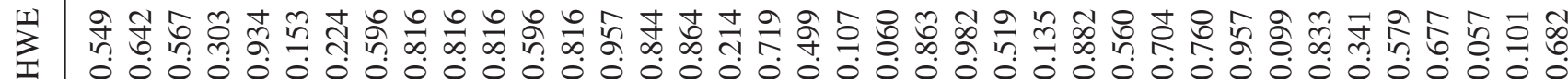

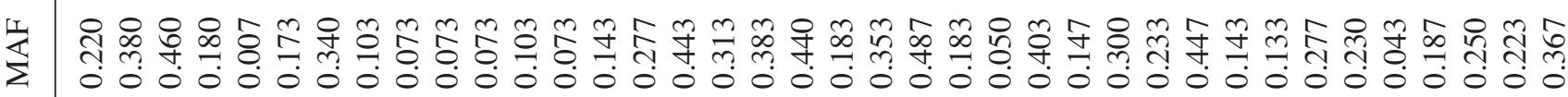

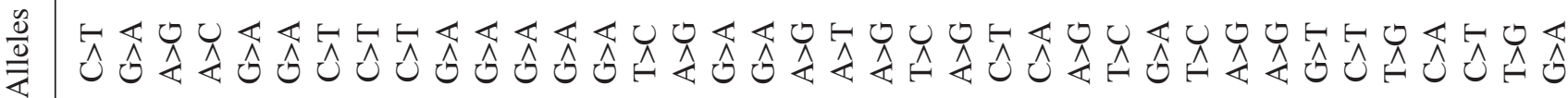

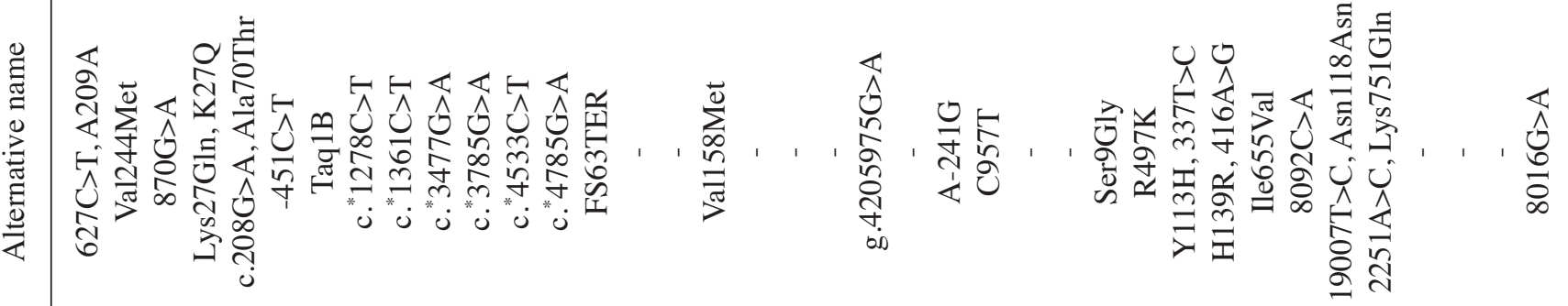




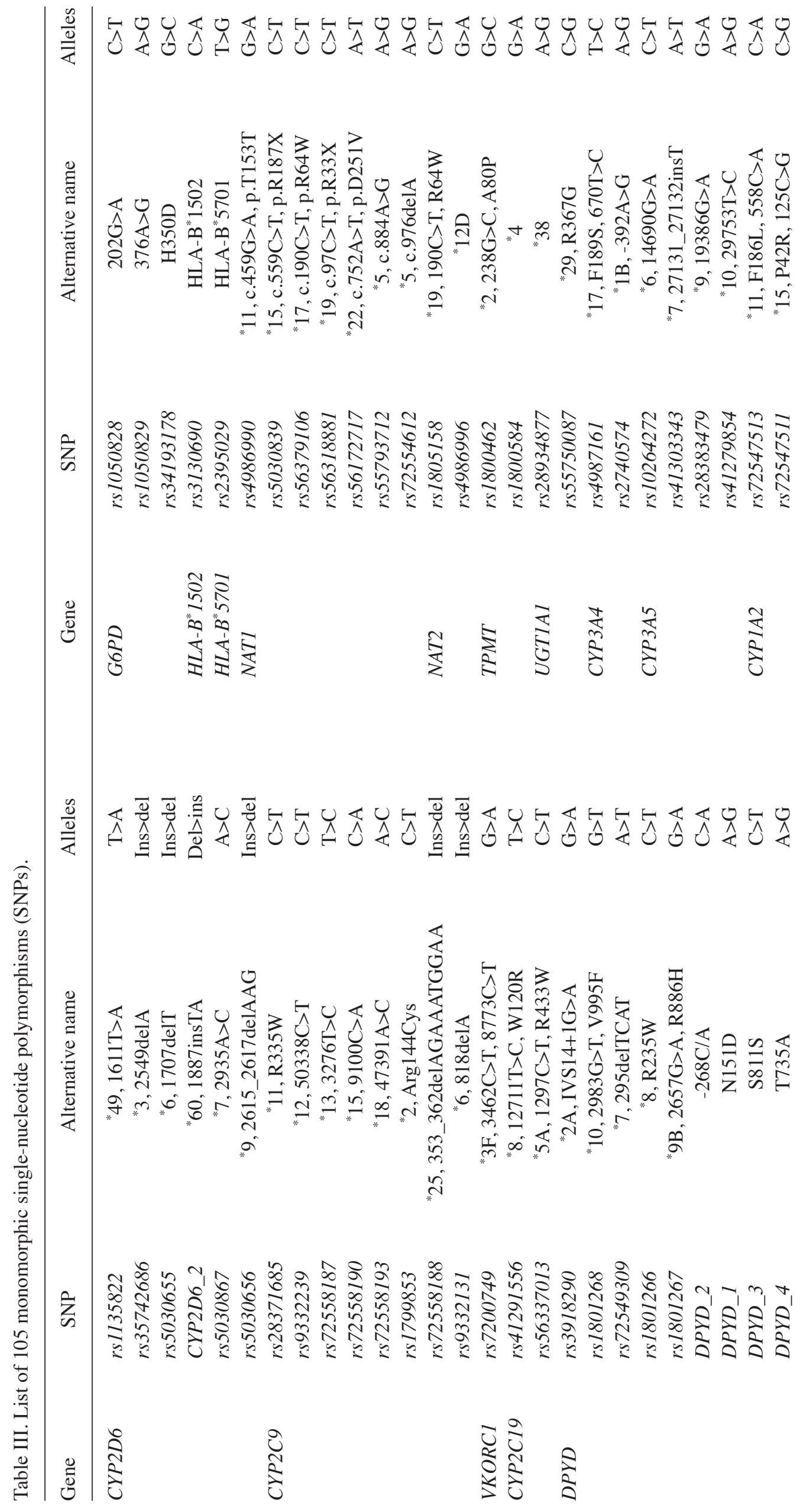




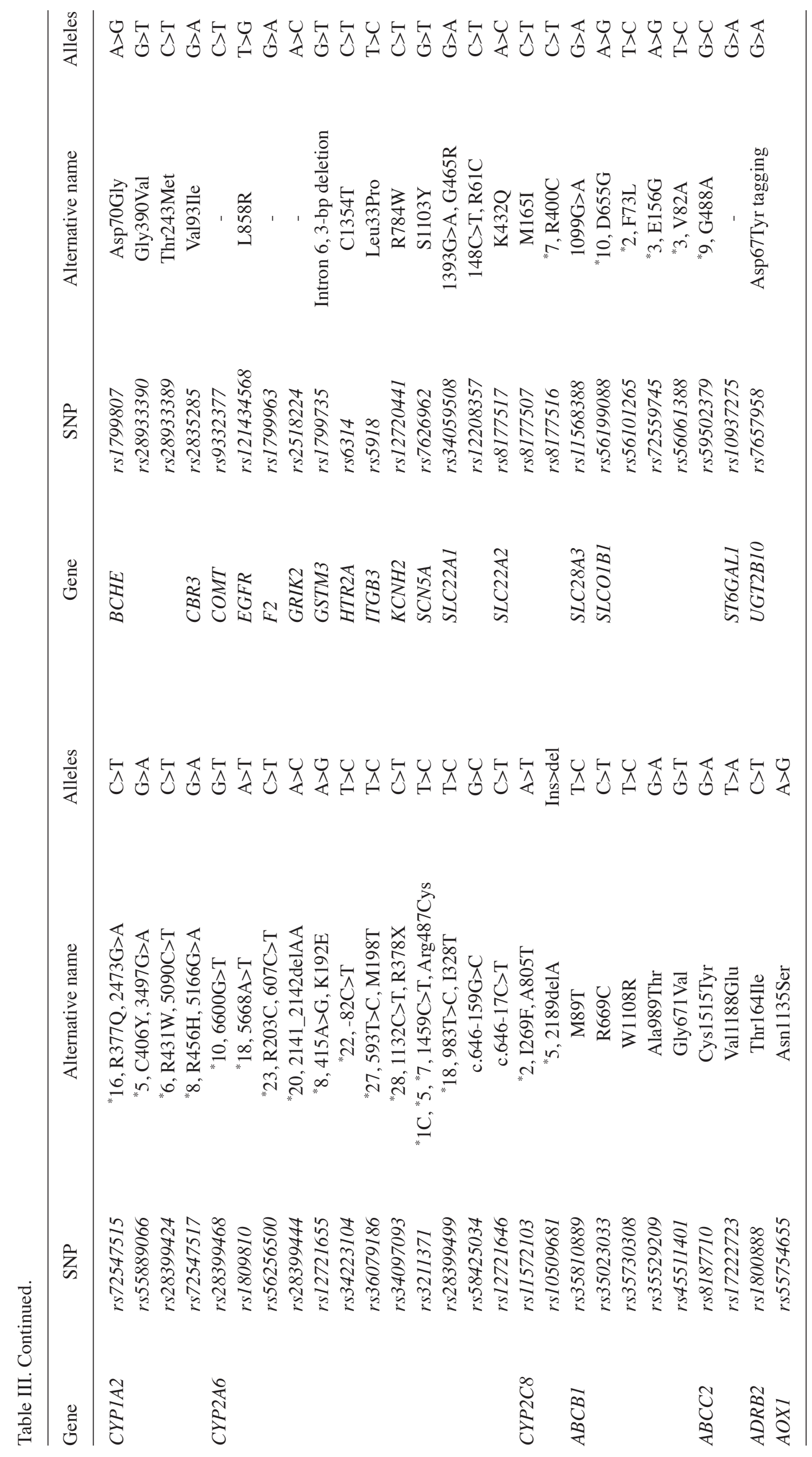




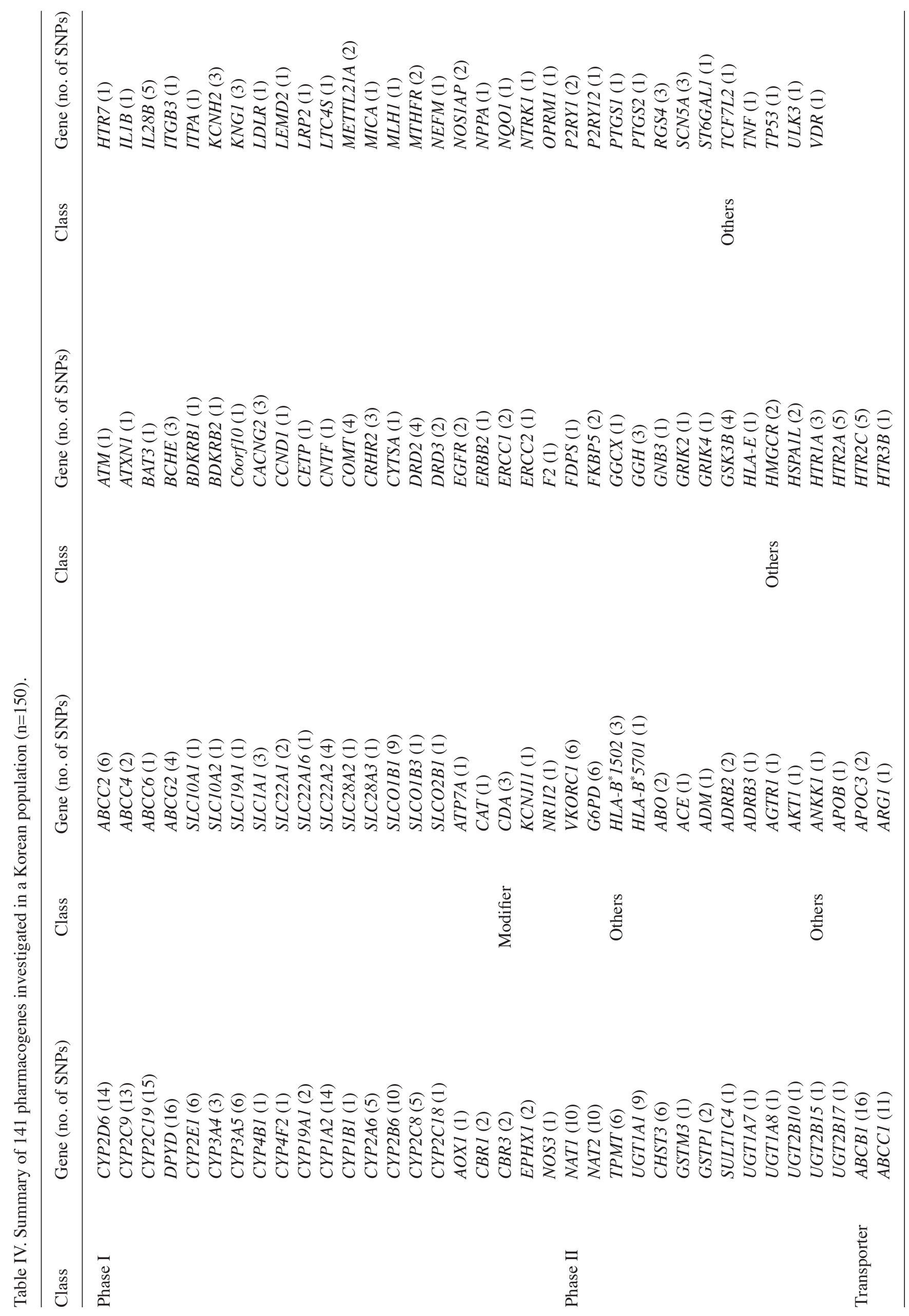




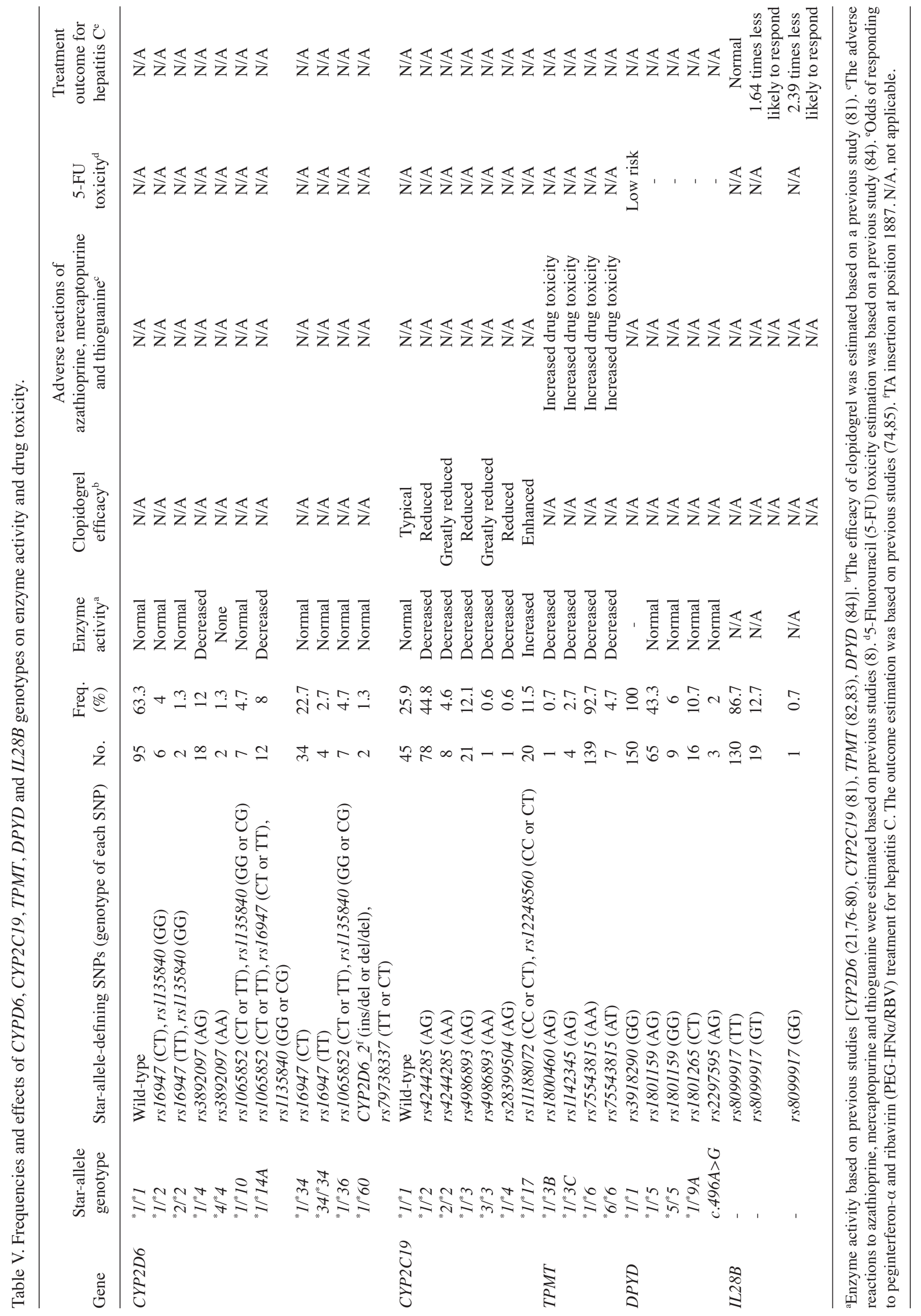


Table VI. Frequencies and effect of combined $C Y P 2 C 9$ and VKORC1 genotypes on the response to warfarin.

\begin{tabular}{|c|c|c|c|c|c|c|c|c|c|c|c|}
\hline & & & \multicolumn{9}{|c|}{ VKORC1 (rs8050894) } \\
\hline \multicolumn{3}{|c|}{ CYP2C9 } & \multicolumn{3}{|c|}{$\mathrm{CC}$} & \multicolumn{3}{|c|}{ CG } & \multicolumn{3}{|c|}{ GG } \\
\hline $\begin{array}{l}\text { Star- } \\
\text { allele } \\
\text { genotype }\end{array}$ & $\begin{array}{c}\text { Star-allele- } \\
\text { defining } \\
\text { SNPs }\end{array}$ & $\begin{array}{l}\text { Genotype } \\
\text { in each } \\
\text { SNP }\end{array}$ & $\mathrm{n}$ & Freq. (\%) & $\begin{array}{l}\text { Warfarin } \\
\text { dose } \\
(\mathrm{mg} / \text { day })^{\mathrm{a}}\end{array}$ & $\mathrm{n}$ & Freq. $(\%)$ & $\begin{array}{l}\text { Warfarin } \\
\text { dose } \\
(\mathrm{mg} / \text { day })^{\mathrm{a}}\end{array}$ & $\mathrm{n}$ & Freq. $(\%)$ & $\begin{array}{l}\text { Warfarin } \\
\text { dose } \\
(\mathrm{mg} / \text { day })^{\mathrm{a}}\end{array}$ \\
\hline${ }^{*} 1 /{ }^{*} 1$ & $\begin{array}{l}r s 1799853 \\
r s 1057910\end{array}$ & $\begin{array}{l}\mathrm{CC} \\
\mathrm{AA}\end{array}$ & 1 & 0.7 & $5-7$ & 22 & 14.7 & $5-7$ & 114 & 76.0 & $3-4$ \\
\hline$* 1 / 2$ & $\begin{array}{l}r s 1799853 \\
r s 1057910\end{array}$ & $\begin{array}{l}\text { CT } \\
\text { AA }\end{array}$ & 0 & 0 & $5-7$ & 0 & 0 & $3-4$ & 0 & 0 & $3-4$ \\
\hline$* 1 / 3$ & $\begin{array}{l}r s 1799853 \\
r s 1057910\end{array}$ & $\begin{array}{l}\mathrm{CC} \\
\mathrm{AC}\end{array}$ & 0 & 0 & $3-4$ & 1 & 0.7 & $3-4$ & 12 & 8.0 & $0.5-2$ \\
\hline$* 2 / 2$ & $\begin{array}{l}r s 1799853 \\
r s 1057910\end{array}$ & $\begin{array}{l}\mathrm{TT} \\
\mathrm{AA}\end{array}$ & 0 & 0 & $3-4$ & 0 & 0 & $3-4$ & 0 & 0 & $0.5-2$ \\
\hline$* 2 / 3$ & $\begin{array}{l}r s 1799853 \\
\text { rs } 1057910\end{array}$ & $\begin{array}{l}\mathrm{CT} \text { or TT } \\
\mathrm{AC} \text { or } \mathrm{CC}\end{array}$ & 0 & 0 & $3-4$ & 0 & 0 & $0.5-2$ & 0 & 0 & $0.5-2$ \\
\hline$* 3 / 3$ & $\begin{array}{l}r s 1799853 \\
r s 1057910\end{array}$ & $\begin{array}{l}\mathrm{CC} \\
\mathrm{CC}\end{array}$ & 0 & 0 & $0.5-2$ & 0 & 0 & $0.5-2$ & 0 & 0 & $0.5-2$ \\
\hline
\end{tabular}

${ }^{a}$ Warfarin dose estimation was based on previous studies $(52,86,87)$.

Table VII. Frequencies and combined effects of $r s 2844682$ and $r s 3909184$ (tagging $H L A-B^{*} 1502$ ) on adverse reactions to carbamazepine.

\begin{tabular}{|c|c|c|c|c|c|}
\hline \multicolumn{2}{|c|}{ SNP/Genotype } & \multirow[b]{2}{*}{$\mathrm{n}$} & \multirow[b]{2}{*}{ Freq. $(\%)$} & \multirow[b]{2}{*}{ HLA-B* 1502 type } & \multirow{2}{*}{$\begin{array}{l}\text { Adverse reactions } \\
\text { to carbamazepine }\end{array}$} \\
\hline$r s 2844682$ & rs3909184 & & & & \\
\hline $\mathrm{CC}$ & $\mathrm{CC}$ & 95 & 63.3 & None & Low risk \\
\hline $\mathrm{CC}$ & $\mathrm{CG}$ & 10 & 6.7 & None & Low risk \\
\hline $\mathrm{CC}$ & GG & 1 & 0.7 & None & Low risk \\
\hline $\mathrm{CT}$ & $\mathrm{CC}$ & 37 & 24.7 & None & Low risk \\
\hline $\mathrm{CT}$ & $\mathrm{CG}$ & 4 & 2.7 & Unable to be determined & - \\
\hline $\mathrm{CT}$ & GG & 0 & 0 & *1502 (one copy) & High risk \\
\hline TT & $\mathrm{CC}$ & 3 & 2.0 & None & Low risk \\
\hline TT & $\mathrm{CG}$ & 0 & 0 & *1502 (one copy) & High risk \\
\hline $\mathrm{TT}$ & GG & 0 & 0 & *1502 (two copies) & High risk \\
\hline
\end{tabular}

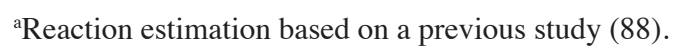

Table VIII. Frequency and effects of HCP5/HLA-B*5701 and TPMT/COMT polymorphisms on adverse drug reaction.

SNP ID/Genotype

\begin{tabular}{|c|c|c|c|c|}
\hline rs2395029 (HCP5) & $H L A-B^{*} 5701$ & $\mathrm{n}$ & Freq. $(\%)$ & Abacavir hypersensitivity $^{\mathrm{a}}$ \\
\hline TT & None & 150 & 100 & Low risk \\
\hline TG & *5701 (one copy) & 0 & 0 & High risk \\
\hline GG & *5701 (two copies) & 0 & 0 & High risk \\
\hline rs1142345 (TPMT) & rs9332377 (COMT) & $\mathrm{n}$ & Freq. $(\%)$ & Adverse reactions to cisplatin ${ }^{\mathrm{b}}$ \\
\hline $\mathrm{AA}$ & $\mathrm{CC}$ & 146 & 93.7 & Low risk \\
\hline $\mathrm{AG}$ & $\mathrm{CC}$ & 4 & 2.7 & High risk \\
\hline GG & $\mathrm{CC}$ & 0 & 0 & High risk \\
\hline
\end{tabular}

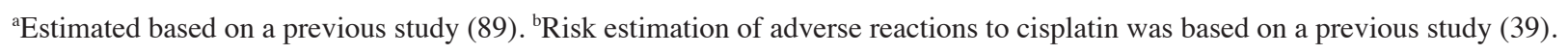


adverse drug reactions or Stevens-Johnson syndrome in Asian populations (47-51). In this study, two tagging SNPs of $H L A-B^{*} 1502$, rs3909184 and $r s 2844682$, were used for the evaluation of the $H L A-B^{*} 1502$ frequency in the Korean population (Table VII). No subject was identified as carrying one or two copies of $H L A-B^{*} 1502$, which is associated with increased risk of adverse reactions to carbamazepine. Thus, the Korean population may have a relatively low risk of adverse reactions to carbamazepine.

$H L A-B^{*} 5701$, which is in linkage disequilibrium with $r s 2395029$ in $H C P 5$, was reported to be a predictive marker of abacavir hypersensitivity (52). Abacavir is an inhibitor of nucleoside reverse-transcriptase and is used as an anti-retroviral agent for human immunodeficiency virus treatment (53). All 150 Korean subjects were found to carry the combination of the TT genotype of $r s 2395029$ and HLA-B*5701-negative type, which is associated with a low risk of hypersensitivity to abacavir (Table VIII). The genotype frequencies of TPMT and catechol O-methyltransferase (COMT) polymorphisms, which are associated with the risk of hearing loss due to cisplatin toxicity (54), were also estimated in the Korean population (Table VIII) and the combination of the AA genotype of $r$ s1142345 (TPMT) and the CC genotype of $r s 9332377$ (COMT), which are associated with a lower risk of cisplatin ototoxicity (54), exhibited the highest frequency (93.7\%) in the Korean population.

In conclusion, we conducted extensive analyses of the distribution of various pharmacogene polymorphisms in 150 Korean subjects and identified the genotype frequencies of important pharmacogene polymorphisms, such as CYP2D6, CYP2C9, VKORC1, CYP2C19, HLA-B and TPMT among others, which may affect the efficacy and side effects of various drugs, including warfarin, clopidogrel, carbamazepine, azathioprine and others. To the best of our knowledge, our study was the first to simultaneously investigate a large number of pharmacogene polymorphisms in multiple samples in a Korean population. The findings from the present study may be helpful in developing personalized medicines for Korean patients. Moreover, the methods used in the present study may also be applied in other populations in order to study their unique pharmacogenomics.

\section{Acknowledgements}

This study was supported by a grant from the Ministry of Food and Drug Safety, Republic of Korea, in 2011 (no. 10182MFDS572).

\section{References}

1. Lazarou J, Pomeranz BH and Corey PN: Incidence of adverse drug reactions in hospitalized patients: a meta-analysis of prospective studies. JAMA 279: 1200-1205, 1998.

2. Dormann H, Neubert A, Criegee-Rieck M, et al: Readmissions and adverse drug reactions in internal medicine: the economic impact. J Intern Med 255: 653-663, 2004.

3. Shastry BS: Pharmacogenetics and the concept of individualized medicine. Pharmacogenomics J 6: 16-21, 2006.

4. Sachidanandam R, Weissman D, Schmidt SC, et al; International SNP Map Working Group: A map of human genome sequence variation containing 1.42 million single nucleotide polymorphisms. Nature 409: 928-933, 2001.

5. Evans WE and Relling MV: Pharmacogenomics: translating functional genomics into rational therapeutics. Science 286 : 487-491, 1999.
6. Evans WE and Johnson JA: Pharmacogenomics: the inherited basis for interindividual differences in drug response. Annu Rev Genomics Hum Genet 2: 9-39, 2001.

7. McLeod HL and Evans WE: Pharmacogenomics: unlocking the human genome for better drug therapy. Annu Rev Pharmacol Toxicol 41: 101-121, 2001.

8. Yates CR, Krynetski EY, Loennechen T, et al: Molecular diagnosis of thiopurine S-methyltransferase deficiency: genetic basis for azathioprine and mercaptopurine intolerance. Ann Intern Med 126: 608-614, 1997.

9. Evans WE and McLeod HL: Pharmacogenomics - drug disposition, drug targets, and side effects. N Engl J Med 348: 538-549, 2003.

10. Johnson JA: Pharmacogenetics: potential for individualized drug therapy through genetics. Trends Genet 19: 660-666, 2003.

11. Guttmacher AE and Collins FS: Welcome to the genomic era. N Engl J Med 349: 996-998, 2003.

12. Zineh I, Gerhard T, Aquilante CL, Beitelshees AL, Beasley BN and Hartzema AG: Availability of pharmacogenomics-based prescribing information in drug package inserts for currently approved drugs. Pharmacogenomics J 4: 354-358, 2004.

13. Schmitz G, Aslanidis C and Lackner KJ: Pharmacogenomics: implications for laboratory medicine. Clin Chim Acta 308: 43-53, 2001.

14. Collins FS, Green ED, Guttmacher AE, Guyer MS, et al: A vision for the future of genomics research. Nature 422: 835-847, 2003.

15. Ragoussis J: Genotyping technologies for genetic research. Annu Rev Genomics Hum Genet 10: 117-133, 2009.

16. Kapoor G, Maitra A and Brahmachari V: Application of SNaPshot for analysis of thiopurine methyltransferase gene polymorphism. Indian J Med Res 129: 500-505, 2009.

17. Fan JB, Gunderson KL, Bibikova M, et al: Illumina universal bead arrays. Methods Enzymol 410: 57-73, 2006.

18. Lin CH, Yeakley JM, McDaniel TK and Shen R: Medium- to high-throughput SNP genotyping using VeraCode microbeads. Methods Mol Biol 496: 129-142, 2009.

19. Sanchez JJ, Borsting C, Hallenberg C, Buchard A, Hernandez A and Morling N: Multiplex PCR and minisequencing of SNPs - a model with 35 Y chromosome SNPs. Forensic Sci Int 137: 74-84, 2003.

20. Hulot JS, Bura A, Villard E, et al: Cytochrome P450 2C19 loss-of-function polymorphism is a major determinant of clopidogrel responsiveness in healthy subjects. Blood 108: 2244-2247, 2006.

21. Savi P, Herbert JM, Pflieger AM, et al: Importance of hepatic metabolism in the antiaggregating activity of the thienopyridine clopidogrel. Biochem Pharmacol 44: 527-532, 1992.

22. Savi P, Combalbert J, Gaich C, et al: The antiaggregating activity of clopidogrel is due to a metabolic activation by the hepatic cytochrome P450-1A. Thromb Haemost 72: 313-317, 1994.

23. Hollopeter G, Jantzen HM, Vincent D, et al: Identification of the platelet ADP receptor targeted by antithrombotic drugs. Nature 409: 202-207, 2001.

24. Derijks LJ, Gilissen LP, Engels LG, et al: Pharmacokinetics of 6-thioguanine in patients with inflammatory bowel disease. Ther Drug Monit 28: 45-50, 2006.

25. Heneghan MA, Allan ML, Bornstein JD, Muir AJ and Tendler DA: Utility of thiopurine methyltransferase genotyping and phenotyping, and measurement of azathioprine metabolites in the management of patients with autoimmune hepatitis. J Hepatol 45: 584-591, 2006.

26. Roman M, Cabaleiro T, Ochoa D, et al: Validation of a genotyping method for analysis of TPMT polymorphisms. Clin Ther 34: 878-884, 2012

27. Hakooz N, Arafat T, Payne D, et al: Genetic analysis of thiopurine methyltransferase polymorphism in the Jordanian population. Eur J Clin Pharmacol 66: 999-1003, 2010.

28. Amstutz U, Froehlich TK and Largiader CR: Dihydropyrimidine dehydrogenase gene as a major predictor of severe 5-fluorouracil toxicity. Pharmacogenomics 12: 1321-1336, 2011.

29. Meyerhardt JA and Mayer RJ: Systemic therapy for colorectal cancer. N Engl J Med 352: 476-487, 2005.

30. Ezzeldin H and Diasio R: Dihydropyrimidine dehydrogenase deficiency, a pharmacogenetic syndrome associated with potentially life-threatening toxicity following 5-fluorouracil administration. Clin Colorectal Cancer 4: 181-189, 2004.

31. van Kuilenburg AB, Maring JG, Schalhorn A, et al: Pharmacokinetics of 5-fluorouracil in patients heterozygous for the IVS14+1G $>$ A mutation in the dihydropyrimidine dehydrogenase gene. Nucleosides Nucleotides Nucleic Acids 27: 692-698, 2008 
32. Suppiah V, Moldovan M, Ahlenstiel G, et al: IL28B is associated with response to chronic hepatitis $\mathrm{C}$ interferon-alpha and ribavirin therapy. Nat Genet 41: 1100-1104, 2009.

33. Tanaka Y, Nishida N, Sugiyama M, et al: Genome-wide association of IL28B with response to pegylated interferon-alpha and ribavirin therapy for chronic hepatitis C. Nat Genet 41: 1105-1109, 2009.

34. Rauch A, Kutalik Z, Descombes P, et al: Genetic variation in IL28B is associated with chronic hepatitis C and treatment failure: a genome-wide association study. Gastroenterology 138: $1338-1345,2010$

35. Glurich I, Burmester JK and Caldwell MD: Understanding the pharmacogenetic approach to warfarin dosing. Heart Fail Rev 15: 239-248, 2010.

36. Schalekamp T, Brasse BP, Roijers JF, et al: VKORC1 and CYP2C9 genotypes and phenprocoumon anticoagulation status: interaction between both genotypes affects dose requirement Clin Pharmacol Ther 81: 185-193, 2007.

37. Wadelius M, Chen LY, Eriksson N, et al: Association of warfarin dose with genes involved in its action and metabolism. Hum Genet 121: 23-34, 2007.

38. Sconce EA, Khan TI, Wynne HA, et al: The impact of CYP2C9 and VKORC1 genetic polymorphism and patient characteristics upon warfarin dose requirements: proposal for a new dosing regimen. Blood 106: 2329-2333, 2005.

39. D'Andrea G, D'Ambrosio RL, Di Perna P, et al: A polymorphism in the VKORC1 gene is associated with an interindividual variability in the dose-anticoagulant effect of warfarin. Blood 105 : 645-649, 2005

40. Schalekamp T, Brasse BP, Roijers JF, et al: VKORC1 and CYP2C9 genotypes and acenocoumarol anticoagulation status: interaction between both genotypes affects overanticoagulation. Clin Pharmacol Ther 80: 13-22, 2006.

41. Wang D, Chen H, Momary KM, Cavallari LH, Johnson JA and Sadee W: Regulatory polymorphism in vitamin K epoxide reductase complex subunit 1 (VKORC1) affects gene expression and warfarin dose requirement. Blood 112: 1013-1021, 2008.

42. Limdi NA, Arnett DK, Goldstein JA, et al: Influence of CYP2C9 and VKORC1 on warfarin dose, anticoagulation attainment and maintenance among European-Americans and African-Americans. Pharmacogenomics 9: 511-526, 2008.

43. Fuerst D, Parmar S, Schumann C, et al: HLA polymorphisms influence the development of skin rash arising from treatment with EGF receptor inhibitors. Pharmacogenomics 13: 1469-1476, 2012 .
44. Melis R, Lewis T, Millson A, et al: Copy number variation and incomplete linkage disequilibrium interfere with the HCP5 genotyping assay for abacavir hypersensitivity. Genet Test Mol Biomarkers 16: 1111-1114, 2012.

45. Maekawa K, Nishikawa J, Kaniwa N, et al: Development of a rapid and inexpensive assay for detecting a surrogate genetic polymorphism of HLA-B 58:01: a partially predictive but useful biomarker for allopurinol-related Stevens-Johnson syndrome/toxic epidermal necrolysis in Japanese. Drug Metab Pharmacokinet 27: 447-450, 2012.

46. Leeder JS: Mechanisms of idiosyncratic hypersensitivity reactions to antiepileptic drugs. Epilepsia 39 (Suppl 7): S8-S16, 1998.

47. Wu XT, Hu FY, An DM, et al: Association between carbamazepine-induced cutaneous adverse drug reactions and the HLA-B*1502 allele among patients in central China. Epilepsy Behav 19: 405-408, 2010.

48. Hung SI, Chung WH, Liu ZS, et al: Common risk allele in aromatic antiepileptic-drug induced Stevens-Johnson syndrome and toxic epidermal necrolysis in Han Chinese. Pharmacogenomics 11: 349-356, 2010.

49. Chen P, Lin JJ, Lu CS, et al: Carbamazepine-induced toxic effects and HLA-B*1502 screening in Taiwan. N Engl J Med 364: 1126-1133, 2011.

50. Zhang Y, Wang J, Zhao LM, et al: Strong association between HLA-B*1502 and carbamazepine-induced Stevens-Johnson syndrome and toxic epidermal necrolysis in mainland Han Chinese patients. Eur J Clin Pharmacol 67: 885-887, 2011.

51. Wang Q, Zhou JQ, Zhou LM, et al: Association between HLA-B*1502 allele and carbamazepine-induced severe cutaneous adverse reactions in Han people of southern China mainland. Seizure 20: 446-448, 2011.

52. Colombo S, Rauch A, Rotger M,et al: The HCP5 single-nucleotide polymorphism: a simple screening tool for prediction of hypersensitivity reaction to abacavir. J Infect Dis 198: 864-867, 2008.

53. Sanchez-Giron F, Villegas-Torres B, Jaramillo-Villafuerte K, et al: Association of the genetic marker for abacavir hypersensitivity HLA-B*5701 with HCP5 rs2395029 in Mexican Mestizos. Pharmacogenomics 12: 809-814, 2011.

54. Ross CJ, Katzov-Eckert H, Dube MP, et al: Genetic variants in TPMT and COMT are associated with hearing loss in children receiving cisplatin chemotherapy. Nat Genet 41: 1345-1349, 2009. 